\title{
Mycotic pulmonary arterial aneurysms in an intravenous drug user
}

\author{
Laura McLean MD FRCPC, Sat Sharma MD FRCPC, Bruce Maycher MD FRCPC \\ Departments of Internal Medicine and Radiology, \\ University of Manitoba, Winnipeg, Manitoba
}

\begin{abstract}
L McLean, S Sharma, B Maycher. Mycotic pulmonary arterial aneurysms in an intravenous drug user. Can Respir J 1998;5(4):307-311.

A case of mycotic pulmonary artery aneurysm (PAA) in an intravenous drug user in whom resolution occurred with conservative therapy is described. The natural history of PAA is not well described in the literature. Although PAA is potentially fatal, resolution may occur in patients who do not have hemoptysis. Clinical presentation, diagnosis and management of PAA are reviewed.
\end{abstract}

Key Words: Intravenous drug user, Mycotic pulmonary artery aneurysm, Pulmonary artery embolization

\section{Anévrismes de l'artère pulmonaire d'origine mycotique chez un toxicomane}

RÉSUMÉ : On décrit ici un cas d'anévrisme de l'artère pulmonaire d'origine mycotique chez un utilisateur de drogues intraveineuses traité avec succès au moyen d'une thérapeutique conservatrice. L'histoire naturelle de l'anévrisme de l'artère pulmonaire n'est pas bien décrite dans la littérature. Bien que cette affection soit potentiellement fatale, la guérison s'observe chez les patients qui ne présentent pas d'hémoptysie. Le tableau clinique, le diagnostic et le traitement de l'anévrisme de l'artère pulmonaire sont passés en revue ici.
$\mathrm{M}$ ycotic pulmonary artery aneurysm (PAA) is a rare disorder (1); few cases of mycotic PAA in intravenous drug users have been reported in the literature. Most cases were unexpectedly diagnosed on autopsy. The natural history of this disorder is not well known.

We report the case of an intravenous drug user who had bilateral PAA confirmed by infused computed tomography (CT) scan of the thorax and pulmonary angiography. The patient was treated with antibiotics and followed with serial CT scans. With antibiotic therapy alone, resolution of the aneurysms occurred without any complications. We reviewed all previously reported cases of mycotic PAAs in intravenous drug users, and briefly describe the pathophysiology, clinical presentation, diagnosis, prognosis and management of the condition.
The present case adds to our understanding of the natural history of PAA in intravenous drug users. It is the first report in the literature where resolution of bilateral PAA in an intravenous drug user is documented without an invasive therapy.

\section{CASE PRESENTATION}

A 28-year-old male was referred for evaluation of exertional dyspnea and chest pain. About one month before, he had been discharged from another hospital after receiving treatment for Staphylococcus aureus pneumonia and septicemia. The patient had been an intravenous heroin and cocaine user for three years before admission. At initial presentation, he had right-sided chest pain, cough and fever. Chest radiograph showed bilateral pneumonia; blood cultures were positive for methicillin-sensitive $S$ aureus. He was adequately 

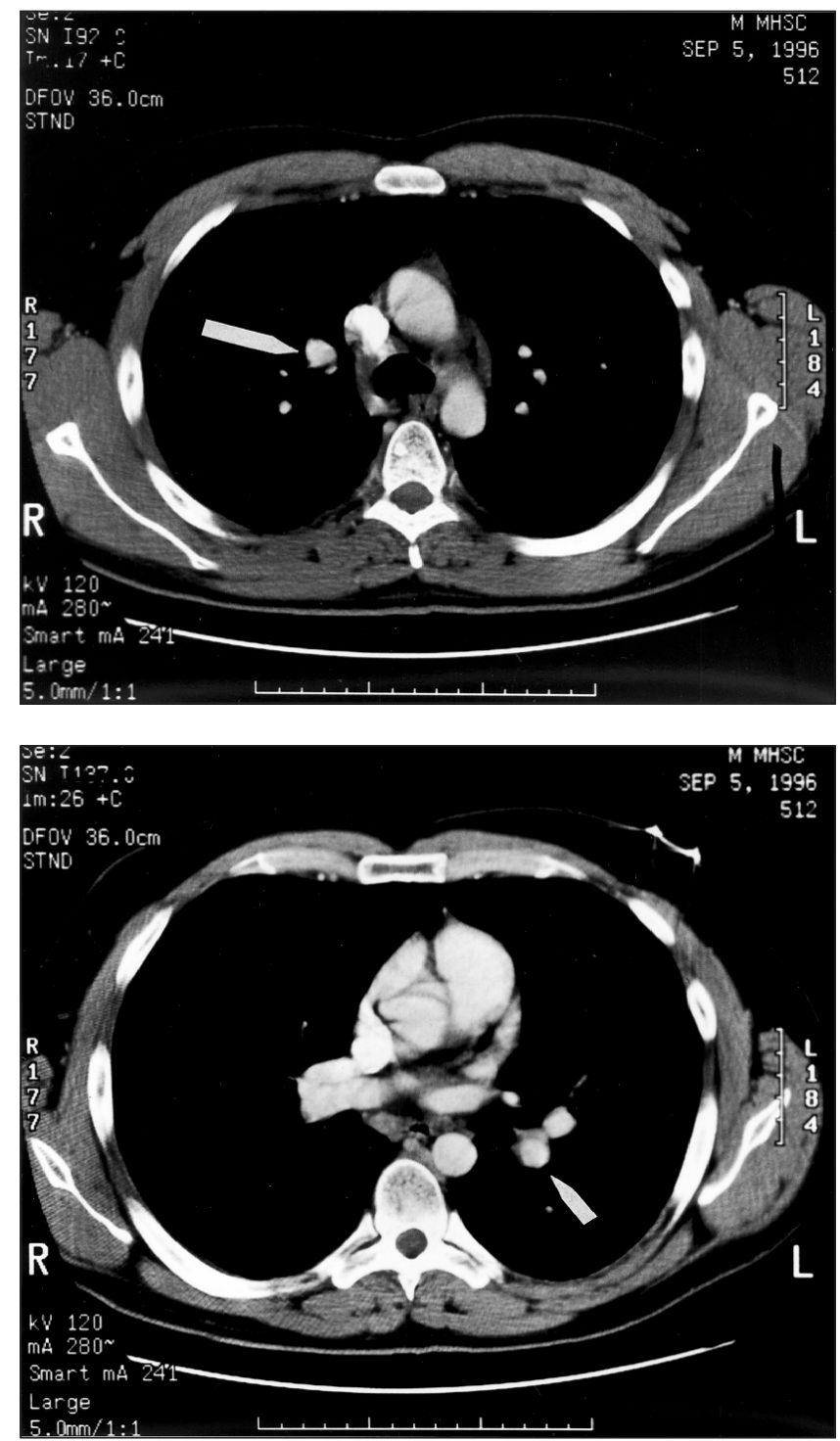

Figure 1) Infused computed tomography of the thorax showing an enhanced nodule superior to the right hilium suggestive of a mycotic aneurysm (top) and occluded lower lobar pulmonary arteries on the left side (bottom)

treated with intravenous cloxacillin and gentamicin for 25 days, followed by oral cloxacillin for two more weeks. An echocardiogram was not completed. Human immunodeficiency virus (HIV) and hepatitis serologies were negative.

He then presented to the St Boniface General Hospital, Winnipeg, Manitoba with mild exertional dyspnea and pleuritic chest pain. He was afebrile, had night sweats and had recently lost $10 \mathrm{~kg}$. He was no longer using intravenous drugs, but occasionally smoked marijuana and consumed alcohol. On examination, he was afebrile, needle track marks were seen on his arms, and respiratory examination revealed decreased breath sounds over the left lower lobe.

Chest radiograph showed patchy areas of consolidation in both lower lobes, a small nodule in the right upper lobe and a nodule in the region of the right hilum. A contrast enhanced CT of the thorax revealed basal pulmonary infarcts bilater-
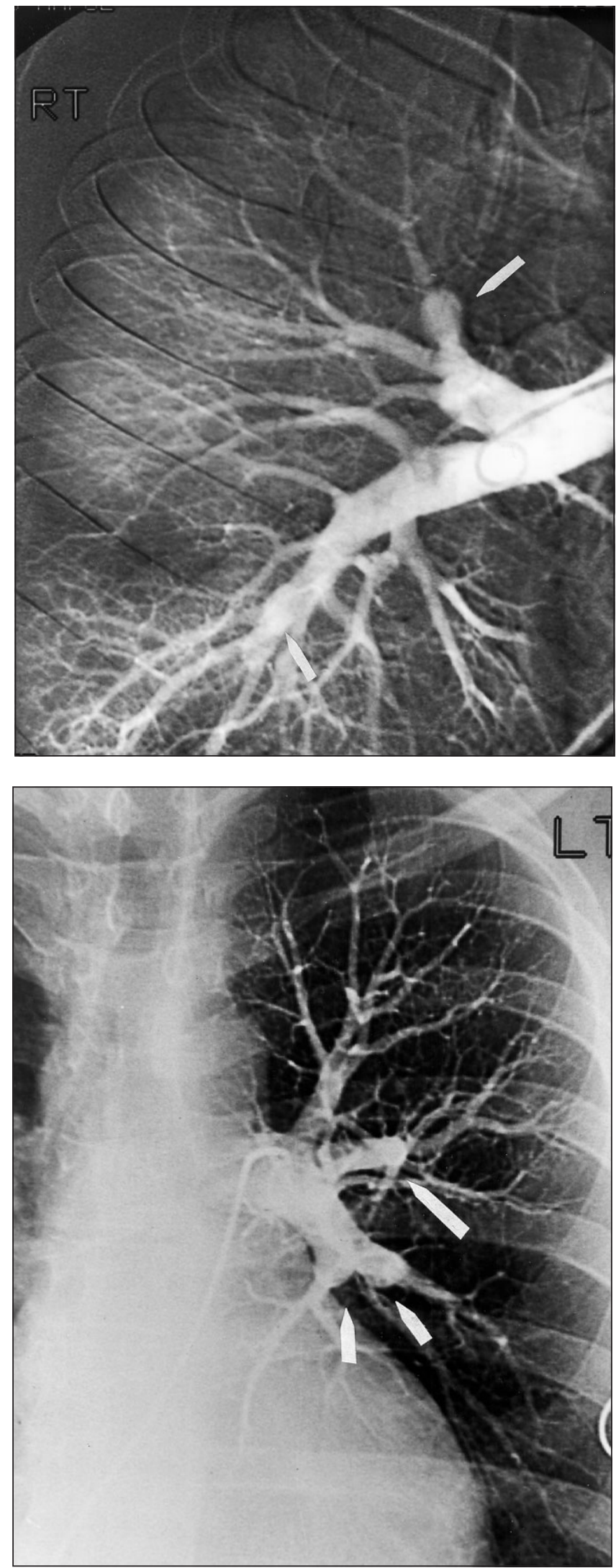

Figure 2) Pulmonary angiogram shows a $1 \mathrm{~cm}$ saccular aneurysm arising from the right apical posterior segmental branch and a fusiform aneurysm in the fight posterobasal segment branch (top). Occulded segmental branches can be seen in the left lower lobe (bottom) 

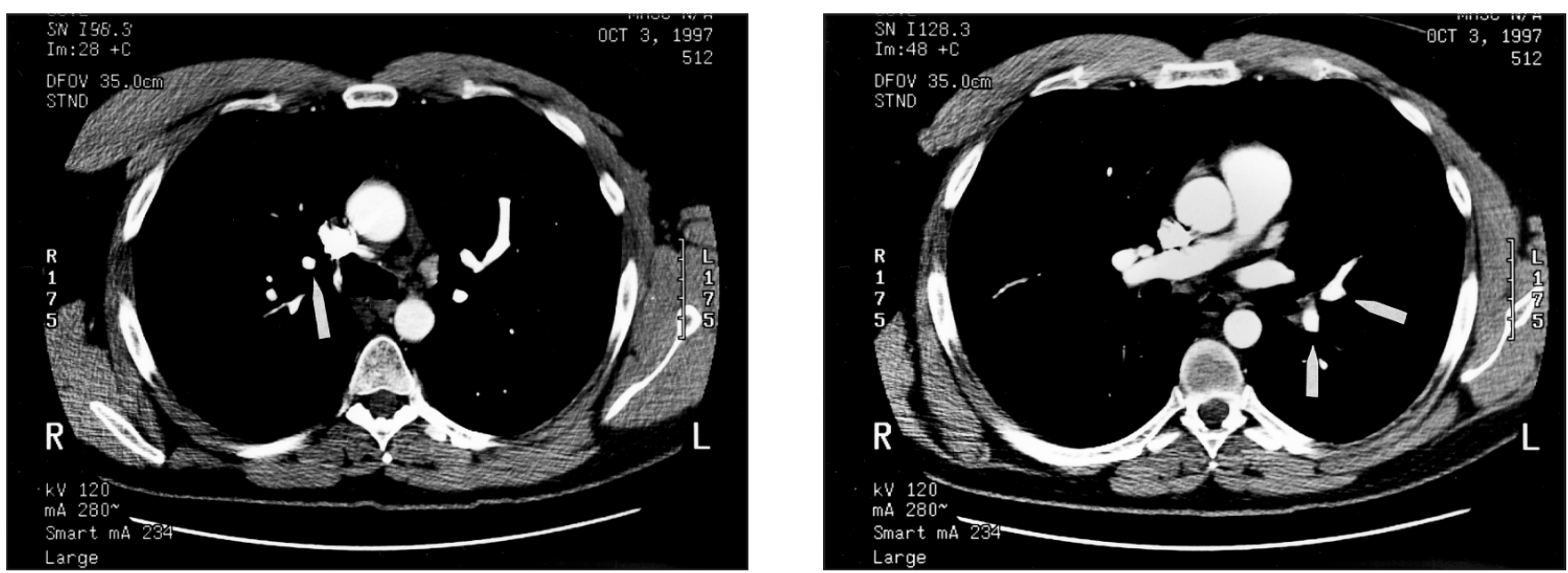

Figure 3) On repeat spiral computed tomography of thorax at 12 weeks, a marked decrease in the size of the PAAs is seen

ally, one with central cavitation, and occluded lobar pulmonary arteries. A small enhancing nodule, superior to the right hilum, suggested the presence of a mycotic aneurysm (Figure 1). A transthoracic echocardiogram did not show evidence of tricuspid valve endocarditis. Duplex ultrasound of the legs was normal.

Pulmonary angiogram showed normal pulmonary artery pressures, a $1 \mathrm{~cm}$ saccular aneurysm arising from the right apical posterior segmental branch and a fusiform aneurysm in the posterobasal segmental branch of the right lower lobe (Figure 2). No aneurysms were seen on the left but occluded segmental branches were noted in the lower lobe. An intraluminal filling defect was not seen in either lung to suggest thromboembolism.

The diagnosis was multiple mycotic pulmonary aneurysms secondary to septic pulmonary emboli, likely from a previous tricuspid endocarditis. The patient declined therapeutic embolization of the pulmonary artery aneurysms, and was followed with serial spiral CT scans every four to six weeks. A repeat spiral CT performed 12 weeks later showed a marked decrease in the size of PAAs (Figure 3).

\section{LITERATURE REVIEW}

Table 1 summarizes the six previously reported cases of mycotic PAA in intravenous drug users and the current case.

The most common presenting symptoms were cough, chest pain, dyspnea, hemoptysis and fever. Of the five patients who reported the type of intravenous drug used, three used amphetamine-like drugs, one used cocaine, and the present patient used both cocaine and heroin. HIV status was unknown in all of the reported cases. All patients had infiltrates or consolidation on chest radiograph. Other common findings were nodules, masses and cavities. Blood cultures were positive in six of the seven cases. All cultures were of Gram-positive organisms, either Staphylococcus or Streptococcus species. None of the reported cases had known congenital heart defects. One patient had pulmonary hypertension (case 3); four of the seven patients had confirmed right-sided endocarditis.
In case 1, a single PAA (size not indicated) was diagnosed on pulmonary angiogram and surgically resected (2). In cases 2,3 and 4, PAAs were found at autopsy (1). The sizes ranged from 0.5 to $3 \mathrm{~mm}$ in diameter, with none of the aneurysms visible on gross inspection. The cause of death in the patients did not appear to be directly related to the aneurysms. In case 5 , pulmonary angiography demonstrated bilateral PAAs of unspecified size (3). These were not felt to be surgically resectable. The patient was lost to follow-up, but resurfaced one and a half years later. A chest radiograph showed diminution in the size of the hilar masses. In case 6, the PAAs were visible grossly at autopsy, size was not indicated and rupture of one of the aneurysms was felt to be the cause of death (4). This patient suffered a massive hemoptysis immediately following bronchoscopy, and died of bleeding into the pleural cavity $56 \mathrm{~h}$ later; PAAs were not diagnosed antemortem.

Of the three patients who had hemoptysis, two died (cases 2 and 6), and the third survived after surgical resection of the aneurysm (case 1).

\section{DISCUSSION}

The association between PAA and intravenous drug use was first noted by Jaffe and Koschmann in 1970 (2). Since then, only seven other cases, including the case described here, have been reported.

The incidence of mycotic PAA in intravenous drug abusers is unknown. A $10 \%$ to $15 \%$ incidence of mycotic PAA as a complication of infective endocarditis has been reported (5). Intravenous drug abusers have an increased incidence of tricuspid valve endocarditis (6).

PAA is most commonly associated with congenital cardiac anomalies such as patent ductus arteriosus, and atrial or ventricular septal defect $(7,8)$. Infection is the second most common cause of PAA (7). Often, PAA appears to result from interplay among two or more of these factors (9).

The pathogenesis of mycotic PAAs secondary to bacterial infection is likely from septic embolism to the pulmonary arteries or vasa vasorum $(9,10)$. An angiocentric pattern of 


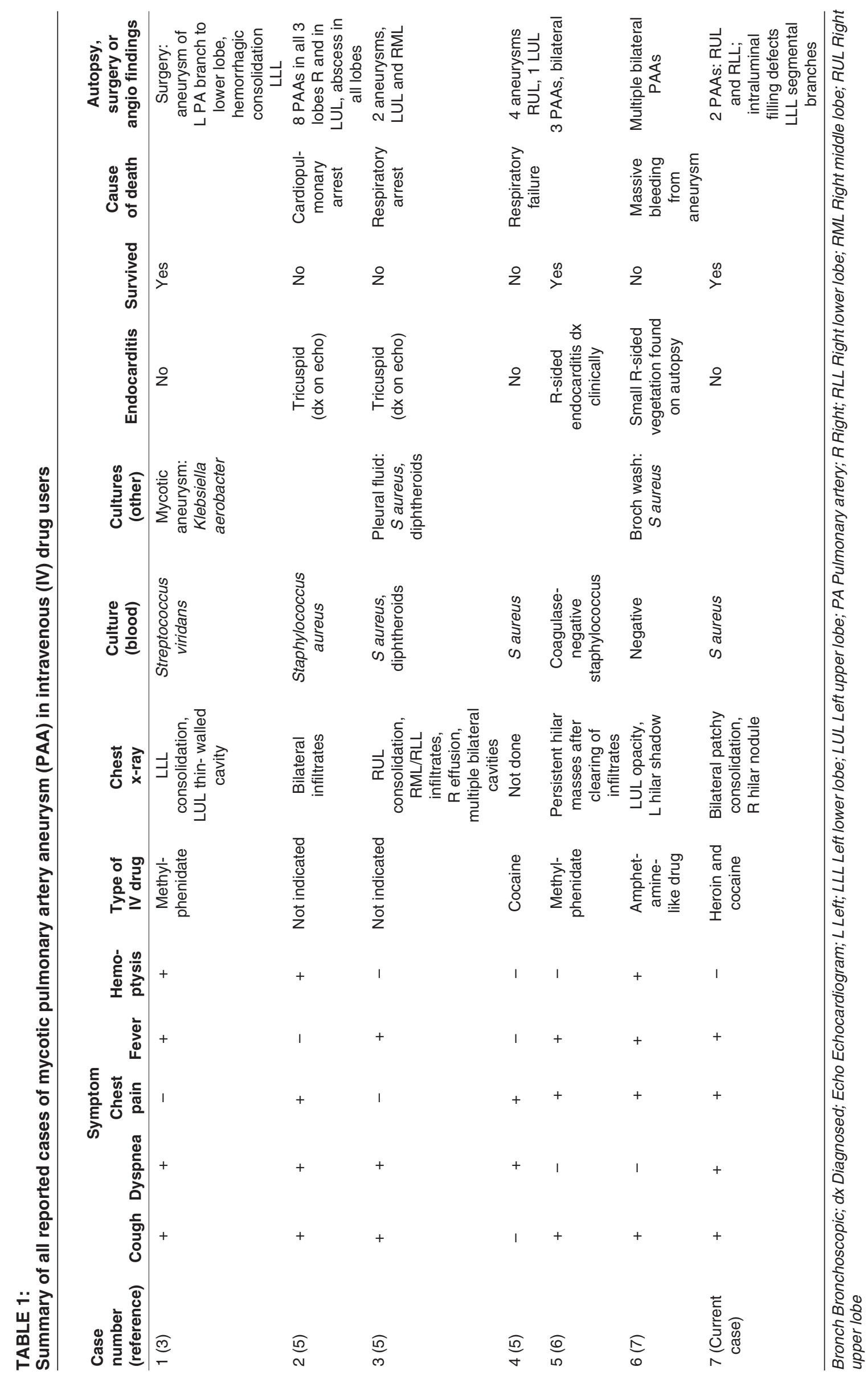


pneumonia may be seen in patients with PAA, consistent with an embolic etiology (1). Direct extension into the vessel from a primary focus of infection in the lung is another possible mechanism $(9,10)$. Destruction of the internal elastic lamina and media occur, and smooth muscle is replaced by fibrous tissue (10). These aneurysms are located peripherally in the pulmonary arterial tree (5). Septic emboli in intravenous drug users would most likely originate from tricuspid vegetations or from septic thrombophlebitis.

In the preantibiotic era, tuberculosis and syphilis were major causes of mycotic PAAs (9). Tuberculous PAAs or Rasmussen's aneurysms arise in vessels that run tangential to tuberculous cavities (9). The vessel wall is replaced by granulomatous tissue that progresses from the outer vessel wall towards the lumen (9). The aneurysms tend to have a peripheral distribution (9). Syphilitic PAAs are found more commonly in a central location (9). Infection of the vasa vasorum, leading to weakening of the vessel wall, and atherosclerosis are the mechanisms by which these aneurysms are formed (9).

To make a diagnosis of mycotic PAA, a high index of suspicion is required. Presenting symptoms are nonspecific, and include cough, dyspnea, chest pain, fever and hemoptysis (9). Physical findings may vary, depending on the etiology of the PAA. In intravenous drug users, evidence of right-sided endocarditis or septic thrombophlebitis may be found. Respiratory examination reveals nonspecific findings such as consolidation or atelectasis.

Chest radiographs may show consolidation, infiltrates, nodular lesions, perihilar masses or cavities. In patients with unresolving pneumonias and mass-like or nodular lesions on chest radiograph, the diagnosis of PAA should be considered. Bronchoscopy and/or inadvertent biopsy of a PAA can lead to massive hemorrhage and death $(4,11)$.

Proximal PAAs may be diagnosed on echocardiography (7). CT with contrast can accurately identify the size and location of PAAs, as can magnetic resonance imaging $(5,12)$. Angiography, however, is felt to be the best diagnostic procedure and can be combined with therapy $(1,9)$. The spiral CT scan has recently emerged as an excellent imaging modality, providing superb visualization of the pulmonary vasculature. The use of spiral CT in evaluation of PAA has not been reported previously. Ours is the first such report where this imaging technology was used to follow the course of PAA.

The natural history of PAAs of any etiology is not well known. It is reported that about one-third of PAAs rupture, causing death $(7,8)$. These lesions are considered to be inherently unstable because any increase in size results in a large increase in wall tension, according to Laplace's law (9). Hemoptysis is felt to be a sign of high risk for rupture and an indication for definitive treatment (7).

The natural history of mycotic PAA in intravenous drug users is unknown; one case was reported where one and a half years after PAA diagnosis on angiography, subsequent chest radiograph showed a decrease in the size of the hilar masses
(3). In our case, progressive decrease in the size of the bilateral PAAs after treatment with antibiotics is well demonstrated with serial infused CT scans. Hemoptysis occurred in neither case.

Treatment for PAAs is directed towards the underlying etiology. In treating mycotic PAA in intravenous drug users, a prolonged course of intravenous antibiotics is required. Obviously, cessation of intravenous drug use is essential. Definitive therapy of PAA can involve surgical or nonsurgical approaches. For proximally located PAAs, surgical repair or pulmonary artery banding is used $(7,9)$. With peripheral PAAs, which are the sites for mycotic PAAs, surgical resection is an option (2,9). But the treatment of choice, especially in patients who have multiple bilateral PAAs or who may not tolerate surgery, is percutaneous catheter embolization of the aneurysm or its feeding vessel, using either detachable balloons or metal coils $(7,9,13)$. Conservative management with antibiotics and a close follow-up to document resolution may be an option in patients who do not have hemoptysis and whose aneurysms are small.

In summary, the incidence or the natural history of mycotic PAAs in intravenous drug users is unknown. Our case suggests that spontaneous resolution of mycotic PAAs in an intravenous drug user may occur following adequate antibiotic therapy.

\section{REFERENCES}

1. Navarro C, Taylor Dickinson PC, Kondlapoodi P, Hagstrom JWC Mycotic aneurysms of the pulmonary arteries in intravenous drug addicts: report of three cases and review of the literature. Am J Med 1984;76:1124-31.

2. Jaffe RB, Koschmann EB. Intravenous drug abuse: pulmonary, cardiac, and vascular complications. Am J Roentgenol 1970;109:107-20.

3. San Dretto MA, Scanlon GT. Multiple mycotic pulmonary aneurysms secondary to intravenous drug abuse. Am J Roentgenol 1984;142:89-90.

4. Morgan JM, Morgan AD, Addis B, Bradley GW, Spiro SG. Fatal haemorrhage from mycotic aneurysms of the pulmonary artery. Thorax 1986;41:70-1.

5. Mootoosamy IM, Reznek RH, Dymond DS, Rees RSO. CT appearance of mycotic aneurysms of the intrapulmonary arteries in the right lung. Br J Radiol 1985;58:85-6.

6. Horsburgh CR Jr, Anderson JR, Boyko EJ. Increased incidence of infections in intravenous drug users. Infect Control Hosp Epidemiol 1989;10:211-5.

7. Luce JM. Pulmonary arteriovenous malformations and other pulmonary vascular abnormalities. In: Murray JF, Nadel JA, eds. Textbook of Respiratory Medicine, 2nd edn. Philadelphia: WB Saunders Company, 1994:1710-24.

8. Gould L, Yang DC, Patel C, et al. Aneurysms of the pulmonary arteries - A case report. Angiology 1987;38:474-8.

9. Bartter T, Irwin RS, Nash G. Aneurysms of the pulmonary arteries. Chest 1988;94:1065-75.

10. Loevner LA, Andrews JC, Francis IR. Multiple mycotic pulmonary artery aneurysms: a complication of invasive mucormycosis. Am J Roentgenol 1992;158:761-2.

11. Roush K, Scala-Barnett DM, Donabedian H, Freimer EH. Rupture of a pulmonary artery mycotic aneurysm associated with candidal endocarditis. Am J Med 1988;84:142-4.

12. Jeang MK, Adyanthaya A, Kuo L, et al. Multiple pulmonary artery aneurysms: new use for magnetic resonance imaging. Am J Med 1986;81:1001-4.

13. Davidoff AB, Udoff EJ, Schonfeld SA. Intra aneurysmal embolization of a pulmonary artery aneurysm for control of hemoptysis. Am J Roentgenol 1984;142:1019-20. 


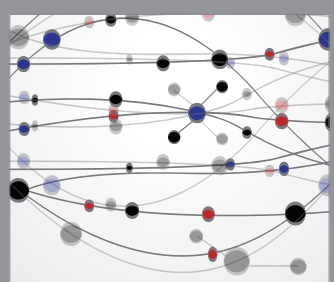

The Scientific World Journal
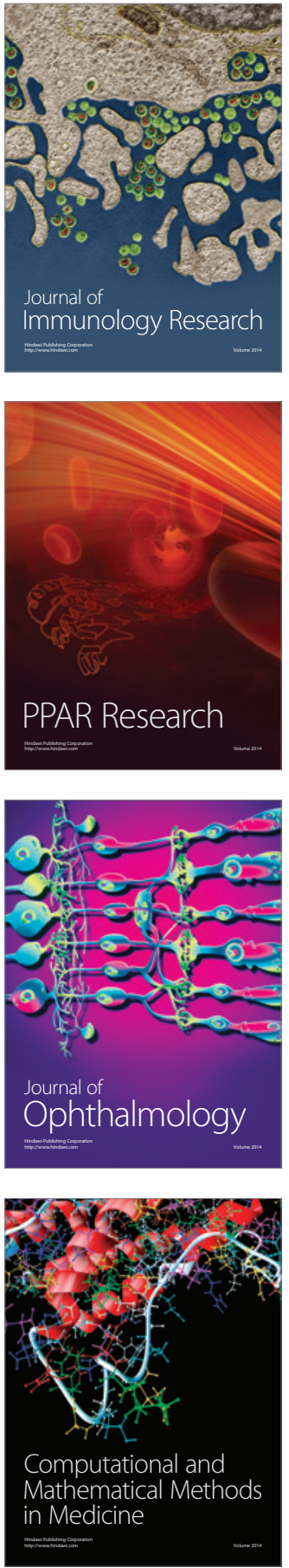

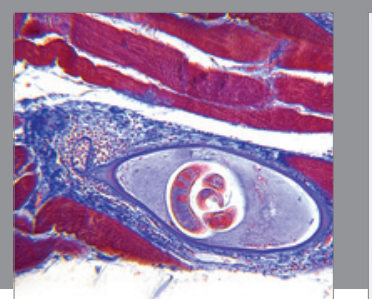

Gastroenterology Research and Practice

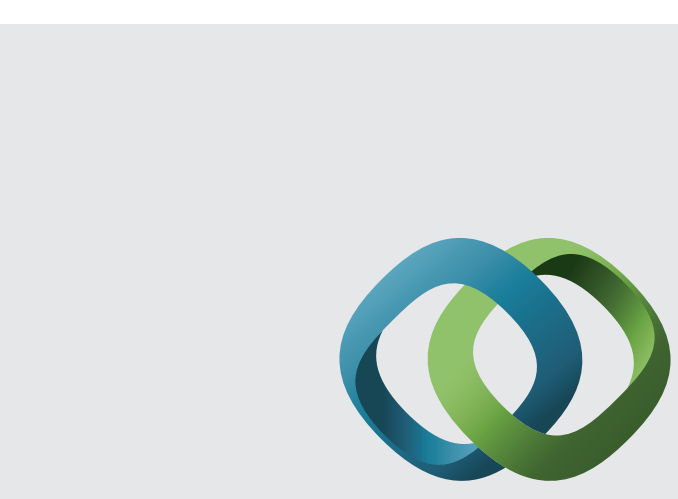

\section{Hindawi}

Submit your manuscripts at

http://www.hindawi.com
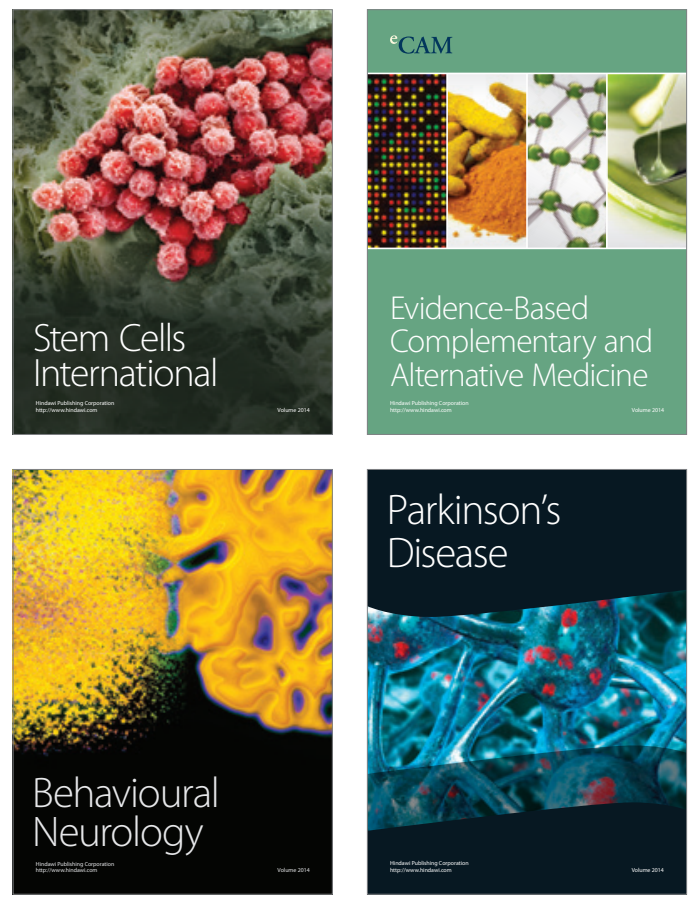
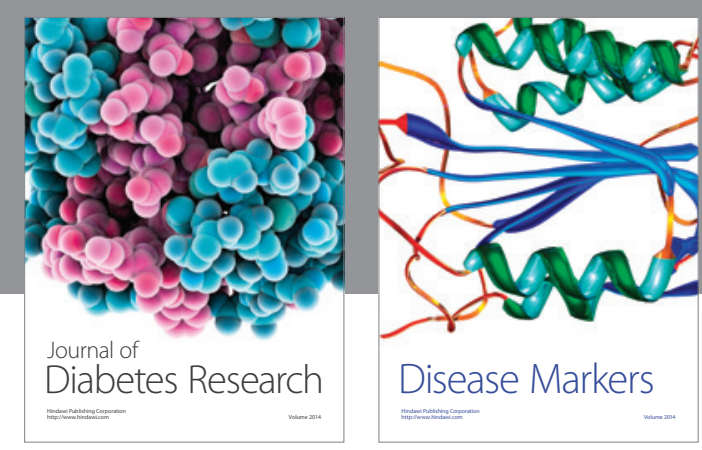

Disease Markers
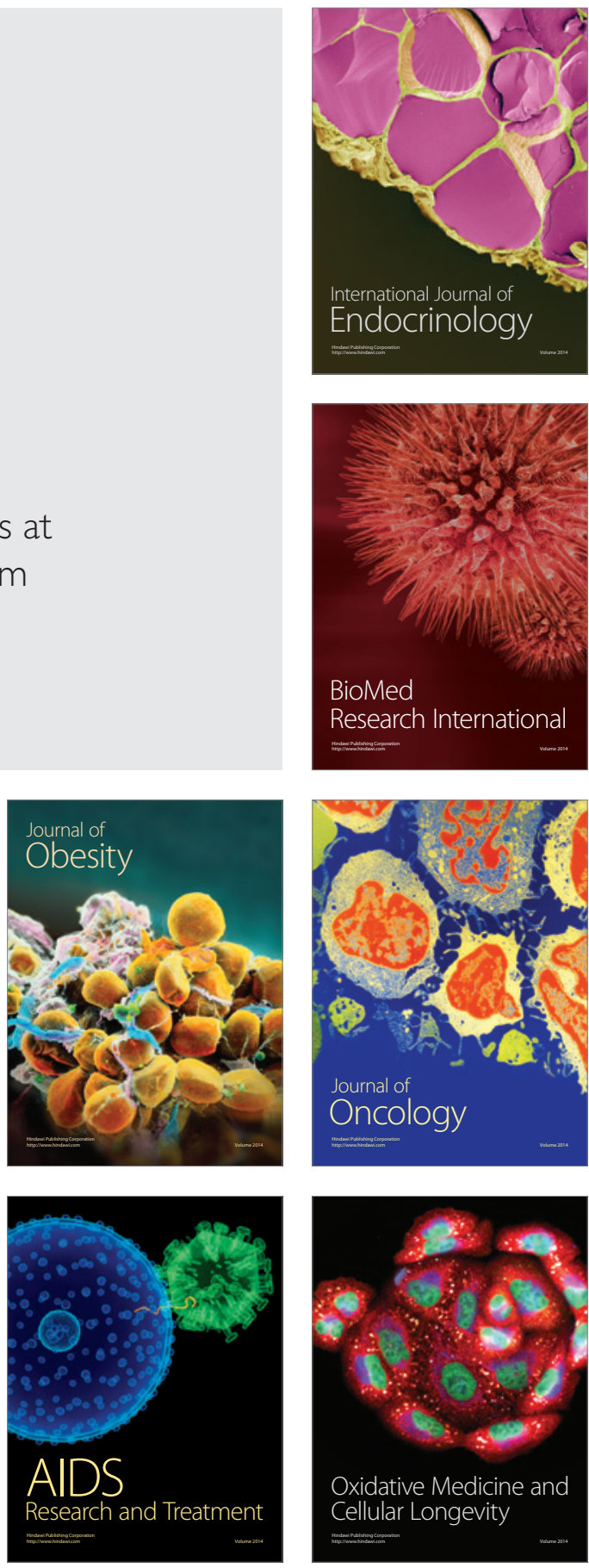